\title{
Should We Look beyond Revised International Prognostic Scoring System: A Retrospective Observational Study of Progression of Myelodysplastic Syndrome to Acute Leukemia
}

\author{
Bangalore Rammohan Nagarjun ${ }^{1}$ Rajashekar Kalaharaghini ${ }^{1}$ Jyoti Sawhney ${ }^{1}$ Pina J. Trivedi ${ }^{1}$ \\ Karthik Dhandapani ${ }^{10}$ Biren Parikh ${ }^{1}$ \\ ${ }^{1}$ Department of OncoPathology, The Gujarat Cancer \& Research \\ Institute, Ahmedabad, Gujarat, India \\ Ind J Med Paediatr Oncol 2021;42:431-438. \\ Address for correspondence Biren Parikh, MD, Department of \\ Oncopathology, The Gujarat Cancer \& Research Institute, Ahmedabad \\ 380016, Gujarat, India (e-mail: birenparikh2002@rediffmail.com).
}

\begin{abstract}
Keywords

- myelodysplastic syndrome

- acute leukemia

- International prognostic scoring system

- cytogenetics

Introduction Myelodysplastic syndrome (MDS) is a clonal stem cell disorder and heterogeneous condition resulting in peripheral cytopenias with marrow dysplasia due to ineffective hematopoiesis. The revised International Prognostic Scoring System (IPSS-R) predicts the risk of progression to acute leukemia (AL). Indian data on MDS and its progression to $\mathrm{AL}$ are limited. Additionally, the cytogenetic findings are dictated by patients' racial background. Study intended to analyze the cytogenetic profile of the patients with MDS.

Objectives This study aimed to (1) evaluate the clinicohematologic and morphologic spectrum of newly diagnosed MDS cases, (2) evaluate the cytogenetic profile of these cases, and (3) study the cases progressed to AL.

Materials and Methods MDS cases diagnosed and followed-up during a 5-year study period, from January 2015 to December 2019 , were included in the study and the study was conducted at regional cancer center in Western India. De novo diagnosed MDS cases with complete workup were considered and MDS due to secondary causes were excluded. Baseline clinical, hematologic findings were tabulated along with cytogenetics and risk stratified as per IPSS-R, and their progression was studied.

Results A total of 63 cases of de novo MDS were diagnosed over a period of 5 years with 45 cases on follow-up and 15 cases (33.3\%) progressed to AL. Maximum number of cases belonged to MDS-EB category accounting to 48 cases (76.1\%). Apparently normal karyotyping was the commonest cytogenetic finding in 33 MDS cases (61.2\%) and in 8 cases that progressed to AL (53.4\%).

Conclusion MDS cases diagnosed at relatively early age were at higher risk of progression to AL. Majority of the cases that progressed to AL were risk stratified in high and very high risk groups and 10 cases which progressed to AL belonged to good category, interestingly apparent normal karyotyping was the commonest cytogenetic
\end{abstract}

DOI https://doi.org/ 10.1055/s-0041-1736175. ISSN 0971-5851.

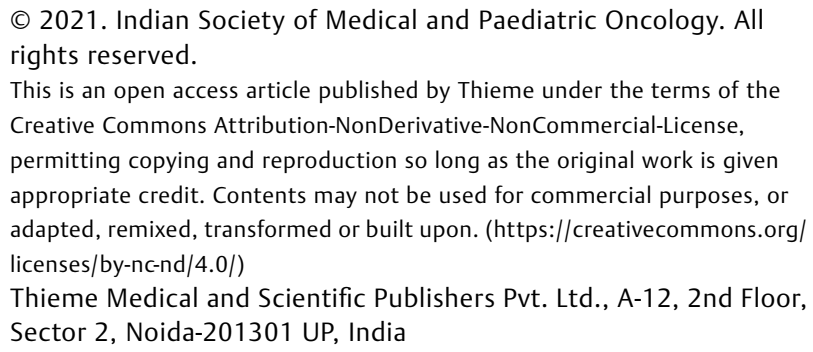

(C) 2021. Indian Society of Medical and Paediatric Oncology. All rights reserved.

This is an open access article published by Thieme under the terms of the Creative Commons Attribution-NonDerivative-NonCommercial-License, permitting copying and reproduction so long as the original work is given appropriate credit. Contents may not be used for commercial purposes, or adapted, remixed, transformed or built upon. (https://creativecommons.org/ licenses/by-nc-nd/4.0/)

Thieme Medical and Scientific Publishers Pvt. Ltd., A-12, 2nd Floor, Sector 2, Noida-201301 UP, India 
finding in more than $50 \%$ of the cases progressed to AL. Molecular mutations could only explain this progression and studies integrating molecular mutations with present IPSS-R scoring system should be conducted, as it could translate into better risk stratification and help in early identification and better management of cases at risk in progression to $\mathrm{AL}$.

\section{Introduction}

Myelodysplastic syndrome (MDS) is a clonal hematological disorder with ineffective hematopoiesis in one or more bone marrow (BM) lineages. ${ }^{1}$ Clinically MDS presents with fatigue, dyspnea, infections, easy bruising due to dysplasia in cell lines, and peripheral cytopenias. BM failure occurs due to ineffective hematopoiesis as a result of excessive apoptosis, maturation arrest, and proliferation resembling the mechanism that play in acute myeloid leukemia (AML). ${ }^{2}$ Multiple factors are implicated in pathophysiology of MDS such as immunologic, cytogenetic, epigenetic, genetic factors, and therapy associated factors. ${ }^{3}$

MDS progress to secondary AML (sAML) in 20 to $30 \%$ of cases, whereas the remaining succumb to progressive $\mathrm{BM}$ failure. ${ }^{4}$ The prognosis of patients transforming to AML is generally grave, as they are resistant to currently available treatment options and the long-term survival rate among treated patients is low. ${ }^{5}$

Owing to the inherent nature of genetic heterogeneity, multiple cytogenetic abnormalities are detected; however, role of each of these in disease progression is not well established. ${ }^{6}$ Risk assessment and therapeutic planning is presently based on the revised International Prognostic Scoring System (IPSS-R). ${ }^{7}$ Cytogenetics (CG), one of the parameters in IPSS-R, is performed mostly by conventional CG. Based on the scoring system, the risk of transformation to AML is assessed and managed accordingly. With advent of sequencing, genetic information obtained can greatly impact the prognosis of the disease. ${ }^{8}$

Deep sequencing studies has facilitated in better understanding of molecular pathogenesis of MDS and its progression. Majority of the mutations do not seem to play any causative role other than genetic instability. ${ }^{6,9}$ Disease progression of MDS is characterized by increasing blast count which is due to acquired additional mutations and genetic alterations in the new emerging clones. ${ }^{9}$ This explains the varied mutational profiles across the different stages of disease.

The model to understand MDS and its progression into AML is a multistep concept of leukemogenesis ${ }^{10,11}$ which is characterized by accumulation of different molecular and genomic alterations belonging to class-I and -II genes and epigenetic modifications $^{12,13}$ that result in the expansion of the MDS/AML clone, this is well understood and accepted.

Spectrum of some mutated genes enriched in SAML is FLT3, NRAS, WT1, IDH1, and IDH2 (type-I genes) which are also the most frequently mutated genes in primary AML. ${ }^{14}$ The commonly mutated genes in high-risk MDS group are
GATA2, RUNX1, TET2, TP53, and ASXL1 (type-II genes). ${ }^{15}$ Leukemic transformation is faster in patients with type-I gene mutations than those without type-I mutations. Epigenetic and RNA splicing genes are commonly mutated in MDS and acquisition of type-I mutations, providing proliferation advantage and explaining the multistep concept of leukemogenesis. ${ }^{16}$

Around 80 to $90 \%$ of MDS cases have somatic mutations identified which determine the clinical phenotype and overall survival. Sequencing studies help to identify mutations which are predictors of poor overall survival. ${ }^{10}$ These mutations are not commonly assessed during patient management as definitive guidelines are not well established. Currently the Indian data on MDS cases and its progression to AL is limited. The study was performed to evaluate clinicohematologic, morphological spectrum, and cytogenetic findings of newly diagnosed MDS along with outcome and also evaluate the cases which progressed to AL with emphasis on their cytogenetic findings.

\section{Materials and Methods}

Observational study was done in a retrospective manner and was conducted in patients diagnosed with MDS at a regional cancer center in western India, from January 2015 to December 2019. Sample size included all the cases diagnosed at our institute over a period of 5 years. Cases which were not evaluated for CG and secondary causes of MDS were excluded. Cases were diagnosed if they met the established criteria, diagnosis of de novo MDS was considered following correlation with morphologic, cytogenetic, and clinical criteria. Cytopenia was diagnosed as per the recommended and established thresholds in IPSS-R (hemoglobin concentration $<10 \mathrm{~g} / \mathrm{dL}$, platelet count $<100 \times 109 / \mathrm{L}$, and absolute neutrophil count $<1.8 \times 10^{9} / \mathrm{L}$ ); BM examination which is considered hallmark in diagnosis of MDS was evaluated for morphologic dysplasia in all the three cell lineages and the established cut-off was $10 \%$ in each lineage to call unilineage or multilineage dysplasia. Dysplastic changes observed in nucleus of erythroid lineage (nuclear budding, internuclear bridging, karyorrhexis, multinuclearity, and megaloblastoid changes) and in cytoplasm (ringed sideroblasts with an aid of Perl's stain and cytoplasmic vacoulations). Dysgranulopoiesis is characterized by small or overtly large cell size, nuclear hypo- or hypersegmentation, decreased granularity, and auer rods. Dysplastic changes in megakaryocytes include micromegakaryocytes, nuclear hypolobation, and multinucleation. Immunohistochemistry was performed to correlate with BM biopsy findings in necessary cases. Biochemical tests such as vitamin B-12, folate, and iron 
profile were performed in all cases to rule out nutritional causes of dysplasia. Any occupational exposure to heavy metals or pesticides and anticancer drugs was excluded. Cases without workup for secondary causes of dysplasia and prior history of treatment for any other malignancy were excluded.

Conventional cytogenetics: BM aspirate sample was sent in heparinized tube for CG. Karyotyping was performed using short-term culture technique and metaphase chromosomes were banded by Giemsa- trypsin- guanine cytosine (GTG) technique and documented as per International System for Human Cytogenetic Nomenclature (ISCN) 2016 guidelines. ${ }^{17}$ At least 20 normal metaphases were necessary to consider a patient cytogenetically normal.

Fluorescence in situ hybridization (FISH): interphase and metaphase FISH was performed and tests were performed as per clinician request. Slides were prepared by phase-contrast microcopy and probes were added to target area of interest. Slides were subjected to denaturation and hybridization process, counterstained with 4',6-diamidino-2-phenylindole (DAPI), and visualized under fluorescence microscope.

FISH was performed with commercially available probes (Vysis), the following probes were used: LSI 5q31 (EGR1) and 7q31 (D7S486). Probe of interest was simultaneously applied (cohybridized) with a different color-labeled probe (internal control). The combinations used were $5 q 31$ (spectrum orange) and D5S721:D5S23 (spectrum green), 7q31 (spectrum orange), and CEP 7 (spectrum green). Cells were considered harboring a gene deletion when carrying two signals due to the internal control and only one signal due to the target sequence and monosomic when carrying one signal each for the internal control and target sequence probes. A total of 200 cells were analyzed and cut-off was $2 \%$ for del $7 q$ and $4 \%$ for del $5 q$.

Diagnosis was based on the World Health Organization (WHO) 2016 criteria and cases prior to this were reevaluated and classified as per the present criteria. With the necessary data, the patients were risk stratified as per IPSS-R system. ${ }^{7}$ During the follow-up period, the cases which progressed to $\mathrm{AL}$ were identified using the WHO determined blast cut-off of $20 \%$.

\section{Statistical Analysis}

Statistical analysis was performed with Statistical Package for Social Science (SPSS) software version 20 (IBM SPSS Statistics for Windows, Armonk, New York, United States; IBM Corp.). Continuous variables were reported as median with standard deviation (SD) and categorical variables as percentages. The association between categorical variables was evaluated for significance, period of progression to $\mathrm{AL}$ was calculated in the each risk group and Student's $t$-test was applied to study the significance of age and its progression also among different risk categories of MDS. The test was considered significant if the $p$-value is $<0.05$.

\section{Results}

Over a period of 5 years, 63 cases of primary MDS were reported (45 men and 18 women) and out of 45 cases on follow-up, 15 cases (33.3\%) progressed to AL during the median follow-up period of 6 months (range: 1-53 months).
Median age at diagnosis of primary MDS is 52 years (range: 11-79 years) and median age of cases which progressed to $\mathrm{AL}$ is 51 years. Among the cases that progressed to $\mathrm{AL}, 53$ years (range: $11-74$ years) was the median age in men (11 cases) and 56 years (range: $51-71$ years) in women (4 cases). Most patients complained of fatigue (58 cases [92.0\%]), breathlessness on exertion (33 cases [52.4\%]), bleeding or easy bruising was presenting complaint (7 seven cases [11.1\%]), six cases (9.5\%) were incidentally detected due to cytopenia/s which was unresponsive to therapy, and two asymptomatic cases (3.1\%) were also referred due to presence of atypical cells/blasts in peripheral blood.

Of the 63 patients, 38 cases (71.9\%) had hemoglobin concentration of $<8 \mathrm{~g} / \mathrm{dL}, 20$ cases $(31.7 \%)$ had between 8 and $10 \mathrm{~g} / \mathrm{dL}$, and 4 cases (6.3\%) had $>10 \mathrm{~g} / \mathrm{dL}$. Mean hemoglobin concentration (MHC) was $7.4 \mathrm{~g} / \mathrm{dL}(\mathrm{SD} \pm 0$ 1.74). Absolute neutrophil count (ANC) less than $800 / \mu \mathrm{L}$ was seen in 21 cases (31.7\%) and remaining case had count more than $800 / \mu \mathrm{L}$. Overall, 31 cases (49.2\%) had platelet count $<50,000 / \mathrm{cmL}, 17$ case $(26.9 \%)$ had between 50,000 and $100,000 / \mathrm{cmL}, 15$ cases (23.8\%) counts were above $100,000 / \mathrm{cmL}$ (-Supplementary Material S1, available in the online version), and 12 cases (19.0\%) had isolated anemia and 29 cases (46.0\%) were pancytopenic. Macrocytic morphology was the commonest finding followed by dimorphic picture and in three cases, microcytic red blood cell (RBC) was noted. Eight cases had blasts in peripheral blood. Vitamin B-12 and serum folate levels evaluated were within normal limits and iron profile in few cases revealed an overload picture.

The distribution of WHO defined MDS entities along with median age in each of the entity is enumerated in (-Table $\mathbf{1}$ ). Maximum number of cases belonged to MDS-excess blast (EB) EB2 category accounted to 32 cases (50.7\%). Cytogenetic study results are available in 54 cases and it includes all cases which progressed to AL. Twenty-one cases (38.9\%) had abnormal karyotype and the CG findings in decreasing order of their prevalence in the study is normal karyotyping (33 cases), del (7q; 8 cases), del (5q; 5 cases), monosomy 7 (3 cases; - Fig. 1) trisomy 8 (3 cases), complex karyotyping $+\operatorname{der}(7) \operatorname{del}(7)$ i( $17 ; 1$ case), and hyperdiploidy ( 1 case; - Table 2 ). Risk groups stratified by IPSS-R and median age in each of the risk groups is tabulated (-Table 3). Forty-five cases were on follow-up and 15 cases (33.3\%) progressed to AL which included one case of acute lymphoblastic leukemia (ALL; - Table 4). Estimated EFS was 5 months (95\% confidence interval [CI]: 0-12 months), 17 cases were on lenalidomide and 5 cases (29.4\%) progressed to AML over 6 months, and 7 out of 13 cases (53.4\%) on hypomethylating agents (HMA) transformed to AML over 6 months. Six cases were on blood transfusion and four cases were on erythropoietin, one case each progressed to AML over 11 and 12 months, respectively. Patient aged 35 years with $15 \%$ blast and marrow fibrosis was treated as AML, 11 months of followup period was uneventful. Hematopoietic stem cell transplantation (HSCT) was performed in an 18-year-old patient belonging high-risk category with normal karyotyping, patient progressed to AML in 48 months and is on follow-up from past 12 months. Patient aged 11 years who progressed to ALL after 4 months of diagnosis of MDS with del $7 \mathrm{q}$ and is on follow-up 
434 MDS Progressing to AL and Looking beyond IPSS-R Nagarjun et al.

Table 1 WHO defined MDS entities with number of cases, median age, cases progressed to AL, and cytogenetic findings in each of the category

\begin{tabular}{|c|c|c|c|c|c|}
\hline \multicolumn{2}{|c|}{$\begin{array}{l}\text { WHO defined } \\
\text { MDS entities }\end{array}$} & $\begin{array}{l}\text { Primary MDS } \\
\text { (63cases) } \\
n(\%)\end{array}$ & $\begin{array}{l}\text { Median age } \\
(y)\end{array}$ & $\begin{array}{l}\mathrm{AL} \\
\text { (15 cases) }\end{array}$ & $\begin{array}{l}\text { Cytogenetic profile } \\
\text { (54 cases) }\end{array}$ \\
\hline \multicolumn{2}{|l|}{ MDS-SLD } & $2(3.2)$ & 60 & 0 & Normal karyotyping \{2\} \\
\hline \multicolumn{2}{|c|}{ MDS-MLD } & $6(9.5)$ & 52.5 & 0 & Del (7q) $\{3\}$, trisomy $8\{1\}$, normal karyotyping $\{1\}$ \\
\hline \multirow[t]{2}{*}{ MDS-RS } & SLD & $1(1.6)$ & 77 & 0 & Trisomy $8\{1\}$ \\
\hline & MLD & $2(3.2)$ & 59 & 0 & Normal karyotyping \{2\} \\
\hline \multicolumn{2}{|c|}{$\begin{array}{l}\text { MDS with isolated } \\
\text { del(5q) }\end{array}$} & $1(1.6)$ & 67 & 0 & $\operatorname{Del}(5 q)\{1\}$ \\
\hline \multirow[t]{2}{*}{ MDS-EB } & EB1 & $16(25.4)$ & 55 & 5 & $\begin{array}{l}\text { Normal karyotyping\{8\},del (5q)\{1\}, del (7q) \{2\}, } \\
\text { hyperdiploidy }\{1\}\end{array}$ \\
\hline & EB2 & $32(50.8)$ & 45.5 & $9+1$ (ALL) & $\begin{array}{l}\text { Normal karyotyping }\{18\} \text {, del }(5 q)\{3\} \text {, del }(7 q)\{3\}, \\
\text { monosomy } 7\{3\} \text {, trisomy } 8\{1\},+\operatorname{der}(7) \operatorname{del}(7) \mathrm{i}(17)\{1\}\end{array}$ \\
\hline \multicolumn{2}{|l|}{ MDS-U } & $3(4.7)$ & 52 & 0 & Normal karyotyping \{2\} \\
\hline
\end{tabular}

Abbreviations: AL, acute leukemia; ALL, acute lymphoblastic leukemia; EB-excess blast; MDS, myelodysplastic syndrome; MLD-multi lineage dysplasia; WHO, World Health Organization; RS- ringed sideroblast; SLD-single lineage dysplasia; U-unclassifiable.

Note: Number of cytogenetic aberration in each subtype is mentioned in within flower brackets.

from past 53 months and is stable. All cases which progressed to AL belonged to MDS-EB category only and majority of them fell into high and very-high-risk IPSS-R groups (- Table 2). Median time taken to progress to AL in high and very high risk groups was 11 and 6 months, respectively, while longer time duration of 16 months was taken by intermediate risk group. Progression to AL in high $(p=0.01)$ and very high risk group $(p=0.002)$ was statistically significant, while intermediate risk group was insignificant $(p=0.3)$. Monosomy 7 belonging to poor cytogenetic risk group, showed $100 \%$ transformation to AL. One case with complex karyotyping was lost for follow-up.

\section{Discussion}

Median age of diagnosis is 51 years and 40 cases (63.5\%) fell in age group less than 60 years which is similar to the Indian study and other Asian studies. ${ }^{18-20}$ Presentation age in Indian population is quite early when compared with the

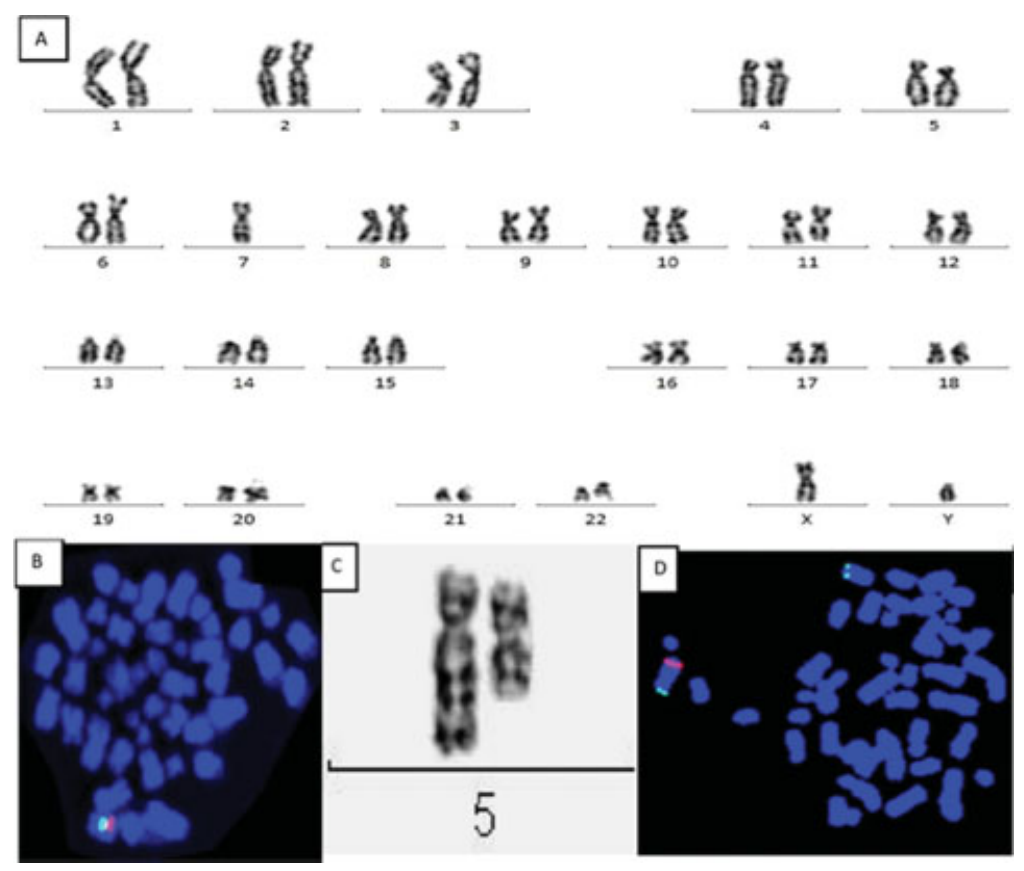

Fig. 1 (A) Complete karyogram shows 45,XY,-7 indicating complete loss of chromosome 7. (B) Metaphase FISH shows orange signal localized to short arm of chromosome 7 (7q31) region and control probe targeting the centromere CEP7 shows green signal. Only one green and orange signal (101G) seen indicates Monosomy 7. (C) Partial karyotype shows interstitial deletion in long arm of chromosome 5. In del(5q), breakpoint occur in q14 and q33. (D) FISH probe localized to short arm of chromosome 5 (5q31) generates orange signal and control probe localized to long arm (5p15.2) and generates green signal. Metaphase FISH shows one orange signal and two green signal (102G) indicating deletion 5q. 
Table 2 Cytogenetic profile of MDS patients and cases progressed to acute leukemia along with median time to progress to $\mathrm{AL}$ in each of the cytogenetics

\begin{tabular}{|l|l|l|l|}
\hline Cytogenetics & $\begin{array}{l}\text { MDS } \\
\text { (54 cases) }\end{array}$ & $\begin{array}{l}\text { MDS cases } \\
\text { progressing to } \\
\text { AL (15 cases) } \\
n \text { (\%) }\end{array}$ & $\begin{array}{l}\text { Median time } \\
\text { for progression } \\
\text { (mo) }\end{array}$ \\
\hline 5q deletion & 5 & $2 / 5(40)$ & 5 \\
\hline Monosomy 7 & 3 & $3 / 3(100)$ & 7 \\
\hline 7q deletion & 8 & $1 / 8(12.5)$ & 3 \\
\hline Trisomy 8 & 3 & $1 / 8(12.5)$ & 1 \\
\hline $\begin{array}{l}\text { Normal } \\
\text { karyotyping }\end{array}$ & 33 & $8 / 33(24.4)$ & 8.5 \\
\hline $\begin{array}{l}\text { Complex } \\
\text { karyotyping }\end{array}$ & 1 & - & - \\
\hline Hyperdiploidy & 1 & - & - \\
\hline
\end{tabular}

Abbreviations: AL, acute leukemia; MDS, myelodysplastic syndrome.

Table 3 Risk stratification of cases based on IPSS-R along with age and median period of progression to $\mathrm{AL}$

\begin{tabular}{|l|l|l|l|l|l|}
\hline IPSS-R & $\begin{array}{l}\text { MDS } \\
(54 \\
\text { cases) } \\
n(\%)\end{array}$ & $\begin{array}{l}\text { Median } \\
\text { age of } \\
\text { MDS (y) }\end{array}$ & $\begin{array}{l}\text { AL } \\
(15 \\
\text { cases) } \\
n(\%)\end{array}$ & $\begin{array}{l}\text { Median } \\
\text { age of } \\
\text { AL (y) }\end{array}$ & $\begin{array}{l}\text { Median } \\
\text { time for } \\
\text { progression } \\
(\mathrm{mo})\end{array}$ \\
\hline Very low & $1(1.5)$ & 26 & - & - & - \\
\hline Low & $4(6.3)$ & 59 & - & - & - \\
\hline Intermediate & $12(19.0)$ & 64.5 & $2(16.7)$ & 65 & 16 \\
\hline High & $21(33.3)$ & 52.0 & $5(23.8)$ & 56 & 11 \\
\hline Very high & $16(25.3)$ & 40.5 & $8(53.3)$ & 53 & 6 \\
\hline
\end{tabular}

Abbreviations: AL, acute leukemia; IPSS-R, revised International Prognostic Scoring System; MDS, myelodysplastic syndrome.

West. ${ }^{21,22}$ Incidence of disease is quite common in men with 45 cases (71.4\%), while 18 cases (28.6\%) in women as established by WHO. ${ }^{7}$ Age at diagnosis is earlier in female group (median age: 43 years) compared with male patients (median age: 54.4 years) and findings were supported by an Indian study. ${ }^{18}$ Most of the cases referred to tertiary cancer center was due to unexplained anemia presenting as persistent fatigue or weakness for which some were transfusion dependent after precluding all treatable causes. Eight cases (12.6\%) were identified through routine checkup. Symptoms due to leucopenia was not observed. Pallor was commonly observed sign. MHC was $7.40 \mathrm{~g} / \mathrm{dL}(\mathrm{SD} \pm 1.74)$. Median age of the patients stratified into very high risk group were younger (40.5 years) with higher risk of progression to AL. Patients with symptomatic anemia were initially supported by blood transfusion and erythropoietin; however, cases with limited response irrespective of del $5 q$ were also treated with lenalidomide. Cases with cytopenias and increased blast count were managed by HMA and during follow-up of 45 cases, 15 cases (33.3\%) progressed to AL. Fourteen cases progressed to AML and one case transformed to ALL. Progression of MDS to AML and, in very rare cases, lymphoblastic type could be explained by hypothesis which states MDS arises from pluripotent hematopoietic stem cell. ${ }^{23}$ It is observed that the elapsed median time to progress to AL is 6 months (range: 1-39).
MDS diagnosed in relatively early age had higher risk for progression to AL. Most of the patients with age group less than 60 years were in high or very high risk category of IPSS$\mathrm{R}(p=0.02)$. Maximum number of cases belonged to MDS-EB category accounting to 48 cases (76.1\%) and 37 cases (68.5\%) belonged to high or very high risk group which is supported by an Asian study which discusses the regional differences in CG. ${ }^{24}$ Cytogenetic information plays crucial role along with degree of dysplasia and blast percentage in risk stratification of de novo MDS by using IPSS-R scoring system and is able to predict the risk of progression to $\mathrm{AL}$ (- Table 3 ). From a total of 15 cases which progressed to AL, 13 cases belonged to high and very high risk groups, but interestingly 10 cases (66.67\%) belonged to good cytogenetic group and consisted of normal karyotyping (eight cases) and del 5q (two cases) and these findings were supported by the Indian study as well. ${ }^{25}$ Progression to AL in cases stratified into high and very high risk groups despite having good cytogenetic subgroup can only be explained by somatic mutations identified by sequencing studies. These mutations also addresses the clinical heterogeneity in the same IPSS-R risk groups with same cytogenetic abnormality. ${ }^{26,27}$

Proportion of abnormal karyotying in our study accounted for 21 cases (38.9\%) and normal karyotying was 33 cases $(61.1 \%)$ which was supported by Kawata et al study and accounted for 36.4 and $63.6 \%$, respectively. ${ }^{28}$ Apparent normal karyotyping is the commonest cytogenetic finding in cases which progressed to $\mathrm{AL}$ (53.4\%) and the reason could be because karyotyping carries inherent limitation of not identifying chromosomal aberration less than $5 \times 10^{6}$ base pairs of DNA, microdeletions, and cryptic translocations. ${ }^{17}$ The study by Kawata et al underscores the importance of gene sequencing, approximately $81 \%$ of cases with normal karyotype revealed mutations which were helpful in better therapeutic decision-making. ${ }^{28}$

Sequencing studies have helped in better understanding of this dynamic and complex nature of MDS and mutations are identified in around 50 genes in 80 to $90 \%$ of MDS cases. ${ }^{16}$ However, the causal relationship of these mutations in AML is not well established. Studies have proven that biological events, such as mutations of type-I genes or epigenetic changes, play driver role in progression to leukemia than chromosomal abnormalities. ${ }^{6}$

Serial sequencing studies help to identify subclones in MDS to sAML which are detected months to years prior to progression become abundant in SAML and also study the clonal evolution during progression to SAML due to selective pressure arising from chemotherapy. Further studies are required to define how to incorporate serial sequencing study results into traditional response criteria in better monitoring of tumor burden. ${ }^{29}$

Currently, there is no consensus on which mutation should be assigned to particular risk category, except for SF3B1 mutation which carries a good prognosis and its association with specific morphology is the only mutation incorporated in WHO classification. Mutations associated with poor prognosis are known; however, their morphological correlation is not well found. ${ }^{30}$ Studies have shown TP53 


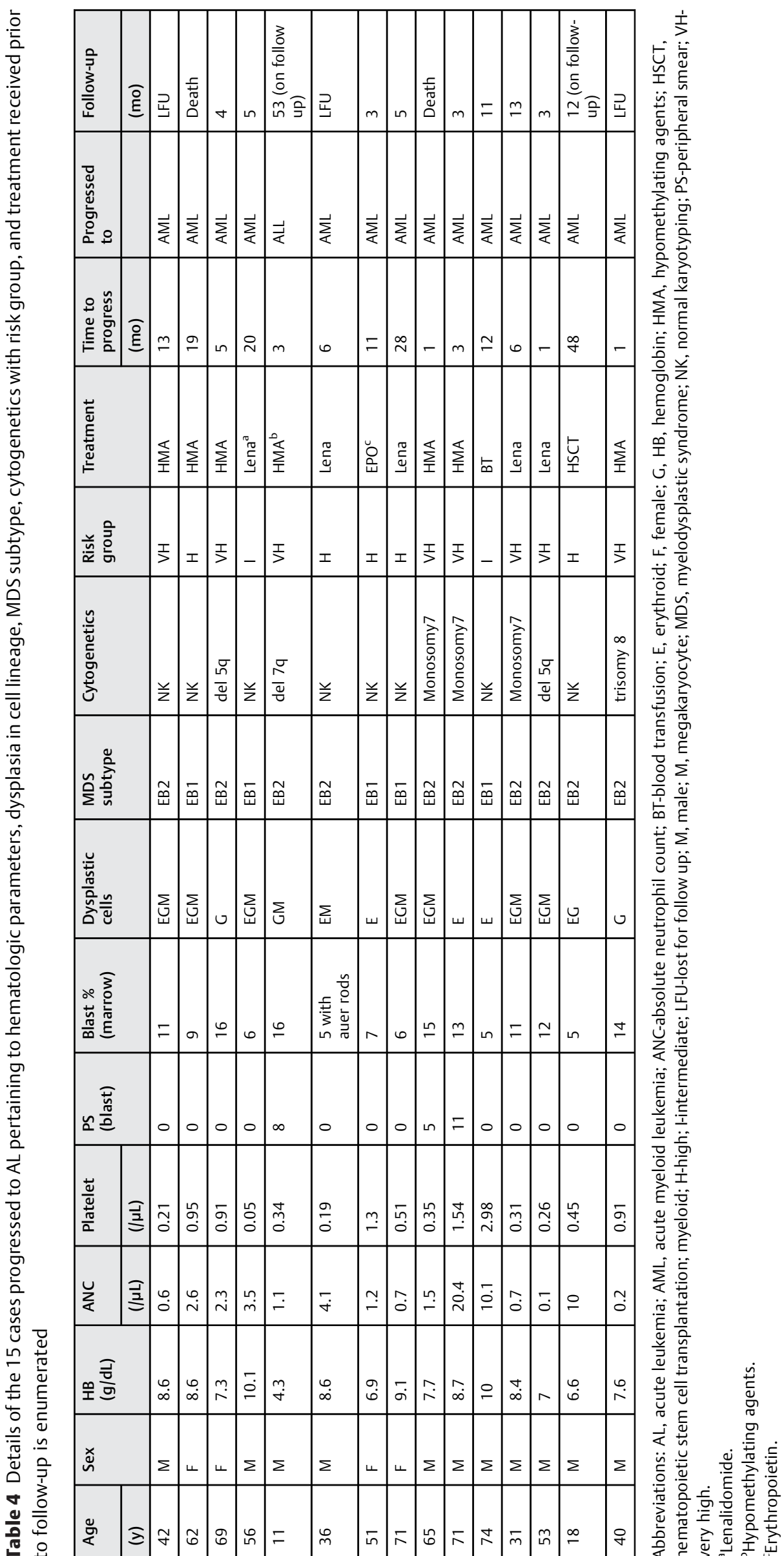


mutation particularly biallelic mutations and specially in complex karyotypes are known to be poor prognostic marker and advocates its incorporation into the scoring system, as it helps in identifying the distinct prognostic subgroups. ${ }^{31,32}$ MDS carries inherent limitation due to heterogeneous nature of the disease; however, laboratory advances have identified genetic mutations and their therapeutic targets and is being translated into personalized medicine in clinical practice. ${ }^{33}$ Sequencing studies are increasingly used in diagnostic practice, validation, and standardization as per the established guidelines increase the reproducibility of the test results. ${ }^{34,35}$ Conducting large multicentric studies with participation of many countries could help in overcoming the racial heterogeneity and the generated high quality sequencing results will help in recognizing significant mutations that can be incorporated into specific risk categories in the prognostic scoring. ${ }^{36,37}$

As modern management strategy, prior identification of somatic mutations along with IPSS-R helps in better risk stratification and can categorize at risk cases. Mutational studies should commonly be performed in all cases, if not feasible at least in cases diagnosed at young age, apparent normal karyotyping, complex karotypes, and in cases which fail to respond to treatment or show signs of clinical deterioration such as increased transfusion dependency or manifestation of proliferative disease. Above-mentioned cytogenetic findings in MDS cases progressed to AL highlight the need for mutational data and its incorporation into the current risk assessment system, as it could offer better insights in risk stratification and in devising specific targeted therapies or transplantation before cases could progress to leukemia.

Serial cytogenetic monitoring was not performed, even in cases which progressed to AL, to assess that the additional cytogenetic finding was the limitation; however, these findings would have limited implications in patient management.

\section{Conclusion}

The study presents the cytogenetic spectrum of MDS cases in western Indian population with special emphasis on its progression to AL. CG continues to be a useful parameter in prognostic scoring. Majority of the cases that progressed to AL were risk stratified in high and very high risk groups and $66.7 \%$ cases had CG belonging to good category, interestingly apparent normal karyotyping was the commonest cytogenetic finding in more than $50 \%$ of the cases that progressed to AL. Mutations could probably explain the reason for progression to AL despite good CG.

\section{Ethics}

The procedures followed were in accordance with the ethical standards of the responsible committee on human experimentation and with the Helsinki Declaration of 1964, as revised in 2013. The Gujarat Cancer \& Research Institute (GCRI) ethical committee board approval was obtained, no: IRC/2021/P-14 on March 19, 2021.
Since the study was retrospective, informed consent was not required and the study did not include any intervention. Waiver of informed consent was obtained from the Ethics Committee.

Conflict of Interest

None declared.

\section{References}

1 Tefferi A, Vardiman JW. Myelodysplastic syndromes. N Engl J Med 2009;361(19):1872-1885

2 Devita VT Jr., Lawrence TA, Rosenberg SA. DeVita, Hellman, and Rosenberg's Cancer: Principles \& Practice of Oncology. 9th ed. Philadelphia, PA: Wolters Kluwer; 2011

3 Visconte V, Selleri C, Maciejewski JP, Tiu RV. Molecular pathogenesis of myelodysplastic syndromes. Transl Med UniSa 2014;8(04): 19-30

4 Steensma DP, Bennett JM. The myelodysplastic syndromes: diagnosis and treatment. Mayo Clin Proc 2006;81(01):104-130

5 Haase D. Cytogenetic features in myelodysplastic syndromes. Ann Hematol 2008;87(07):515-526

6 Shukron O, Vainstein V, Kündgen A, Germing U, Agur Z. Analyzing transformation of myelodysplastic syndrome to secondary acute myeloid leukemia using a large patient database. Am J Hematol 2012;87(09):853-860

7 Swerdlow SH, Campo E, Harris NL, et al. WHO Classification of Tumors of Haematopoietic and Lymphoid Tissues. 4th ed. Lyon, France: IARC; 2017

8 Swoboda DM, Sallman DA. Mutation-driven therapy in MDS. Curr Hematol Malig Rep 2019;14(06):550-560

9 Walter MJ, Shen D, Ding L, et al. Clonal architecture of secondary acute myeloid leukemia. N Engl J Med 2012;366 (12):1090-1098

10 Bejar R, Stevenson K, Abdel-Wahab O, et al. Clinical effect of point mutations in myelodysplastic syndromes. N Engl J Med 2011;364 (26):2496-2506

11 Georgiou G, Karali V, Zouvelou C, et al. Serial determination of FLT3 mutations in myelodysplastic syndrome patients at diagnosis, follow up or acute myeloid leukaemia transformation: incidence and their prognostic significance. $\mathrm{Br}$ J Haematol 2006;134 (03):302-306

12 Parker JE, Mufti GJ, Rasool F, Mijovic A, Devereux S, Pagliuca A. The role of apoptosis, proliferation, and the Bcl-2-related proteins in the myelodysplastic syndromes and acute myeloid leukemia secondary to MDS. Blood 2000;96(12):3932-3938

13 Watanabe-Okochi N, Kitaura J, Ono R, et al. AML1 mutations induced MDS and MDS/AML in a mouse BMT model. Blood 2008; 111(08):4297-4308

14 Lindsley RC, Mar BG, Mazzola E, et al. Acute myeloid leukemia ontogeny is defined by distinct somatic mutations. Blood 2015; 125(09):1367-1376

15 Makishima H, Yoshizato T, Yoshida K, et al. Dynamics of clonal evolution in myelodysplastic syndromes. Nat Genet 2017;49(02): 204-212

16 Ogawa S. Genetics of MDS. Blood 2019;133(10):1049-1059

17 McGowan-Jordan J, Simons A, Schmid MInternational Standing Committee on Human Cytogenomic Nomenclature. ISCN: An International System for Human Cytogenetic Nomenclature (2016). Basel, New York: Karger; 2016:14

18 Chaubey R, Sazawal S, Mahapatra M, Chhikara S, Saxena R. Does Indian myelodysplastic syndrome have a biology different from that in the West? Asian Pac J Cancer Prev 2016;17(04): 2341-2342

19 Lee JJ, Kim HJ, Chung IJ, et al. Comparisons of prognostic scoring systems for myelodysplastic syndromes: a Korean multicenter study. Leuk Res 1999;23(05):425-432 
20 Chen B, Zhao WL, Jin J, et al. Clinical and cytogenetic features of 508 Chinese patients with myelodysplastic syndrome and comparison with those in Western countries. Leukemia 2005;19(05): 767-775

21 Ma X, Does M, Raza A, Mayne ST. Myelodysplastic syndromes: incidence and survival in the United States. Cancer 2007;109(08): 1536-1542

22 Neukirchen J, Schoonen WM, Strupp C, et al. Incidence and prevalence of myelodysplastic syndromes: data from the Düsseldorf MDS-registry. Leuk Res 2011;35(12):1591-1596

23 Algarni AA, Akhtari M, Fu K. Myelodysplastic syndrome with myelofibrosis transformed to a precursor B-cell acute lymphoblastic leukemia: a case report with review of the literature. Case Rep Hematol 2012;2012:207537

24 Jiang Y, Eveillard JR, Couturier MA, et al. Asian population is more prone to develop high-risk myelodysplastic syndrome, concordantly with their propensity to exhibit high-risk cytogenetic aberrations. Cancers (Basel) 2021;13(03):481

25 Chaubey R, Sazawal S, Dada R, Mahapatra M, Saxena R. Cytogenetic profile of Indian patients with de novo myelodysplastic syndromes. Indian J Med Res 2011;134(04):452-457

26 Gondek LP, Tiu R, O’Keefe CL, Sekeres MA, Theil KS, Maciejewski JP. Chromosomal lesions and uniparental disomy detected by SNP arrays in MDS, MDS/MPD, and MDS-derived AML. Blood 2008;111 (03):1534-1542

27 Haase D, Germing U, Schanz J, et al. New insights into the prognostic impact of the karyotype in MDS and correlation with subtypes: evidence from a core dataset of 2124 patients. Blood 2007;110(13):4385-4395

28 Kawata E, Xenocostas A, Hsia CC, et al. Reevaluating the role of cytogenetic testing in patients with suspected myelodysplastic syndrome in the era of next generation sequencing. Blood 2019; 134(Suppl 1):3438

29 Menssen AJ, Walter MJ. Genetics of progression from MDS to secondary leukemia. Blood 2020;136(01):50-60

30 National Comprehensive Cancer Network. Myelodysplastic syndrome (version 2.2020). Accessed August 10, 2021 at: http:// www.nccn.org/professionals/physician_gls/pdf/mds_blocks.pdf

31 Haase D, Stevenson KE, Neuberg D, et al; International Working Group for MDS Molecular Prognostic Committee. TP53 mutation status divides myelodysplastic syndromes with complex karyotypes into distinct prognostic subgroups. Leukemia 2019;33(07):1747-1758

32 Sanz GF, Ibañez M, Such E. Do next-generation sequencing results drive diagnostic and therapeutic decisions in MDS? Blood Adv 2019;3(21):3454-3460

33 Pagliuca S, Gurnari C, Visconte V. Molecular targeted therapy in myelodysplastic syndromes: new options for tailored treatments. Cancers (Basel) 2021;13(04):784

34 Roy S, Coldren C, Karunamurthy A, et al. Standards and guidelines for validating next-generation sequencing bioinformatics pipelines: a joint recommendation of the Association for Molecular Pathology and the College of American Pathologists. J Mol Diagn 2018;20(01):4-27

35 Palomo L, Ibáñez M, Abáigar M, et al; Spanish Group of MDS (GESMD) Spanish Guidelines for the use of targeted deep sequencing in myelodysplastic syndromes and chronic myelomonocytic leukaemia. Br J Haematol 2020;188(05):605-622

36 Du MY, Xu M, Deng J, et al. Evaluation of different scoring systems and gene mutations for the prognosis of myelodysplastic syndrome (MDS) in Chinese population. J Cancer 2020;11(02):508-519

37 Platzbecker UUwePlatzbecker. Treatment of MDS. Blood 2019; 133(10):1096-1107 